\title{
超临界 $\mathrm{CO}_{2}$ 在多孔材料合成中的应用
}

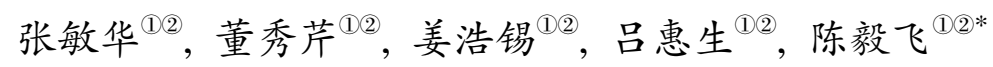

(1) 天津大学绿色合成与转化教育部重点实验室, 石油化工技术开发中心, 天津 300072;

(2) 化学化工协同创新中心, 天津 300072

*联系人, E-mail: tdchenyifei@126.com

2015-03-30 收稿, 2015-04-10 接受, 2015-05-29 网络版发表

国家自然科学基金(20976120)和天津市自然科学基金(09JCYBJC06200)资助

摘要 超临界流体技术作为一种绿色技术在多孔材料合成领域受到了研究者的广泛关注. 与传统方法相比较, 利用超临界 $\mathrm{CO}_{2}$ 制备多孔材料的方法具有方法简单、环保、产品性能好 等优点, 且 $\mathrm{CO}_{2}$ 无毒、价廉、原料易得, 具有很好的工业应用前景. 本文概述了近年来超临 界 $\mathrm{CO}_{2}$ 在分子篎、碳纳米管、金属/复合氧化物纳米粒子、金属有机骨架材料、聚合物等多 孔材料的合成方面的应用和研究进展.

关键词

超临界 $\mathrm{CO}_{2}$ 多孔材料

分子篮

金属有机骨架 金属/复合氧化物 超临界流体(superctitical fluid, SCF)是物质的温 度和压力同时高于其临界值时的流体, 如图 1 所示. SCF兼具液体和气体的优点, 既有接近液体的密度和 溶解能力, 又有接近气体的黏度和扩散性质. 其优异 的物理化学特性使得其在萃取分离、化学反应工程、 材料科学、环境保护、食品、医药、分析技术、微电

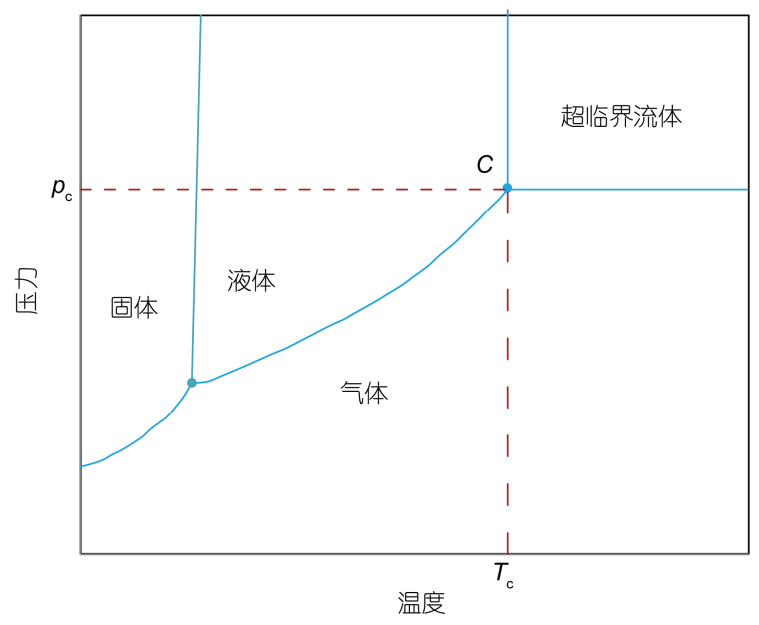

图 1 (网络版彩色)纯物质相图

Figure 1 (Color online) Phase diagram of pure substances
子技术等众多领域显示出良好的应用前景 ${ }^{[1]}$. 其中, 超临界 $\mathrm{CO}_{2}\left(\mathrm{scCO}_{2}\right)$ 具有临界条件 $\left(\mathrm{CO}_{2}\right.$ 的临界温度 $T_{\mathrm{c}}=304.18 \mathrm{~K}$, 临界压力 $P_{\mathrm{c}}=7.38 \mathrm{MPa}$ )温和、性质稳 定、安全性好、价格便宜和可回收利用等优点, 是有 机溶剂的理想替代品, 为“绿色化学”发展不可或缺 的介质, 是目前研究最为广泛的超临界流体. 近年 来, 随着超临界流体技术的迅速发展, $\mathrm{scCO}_{2}$ 在多孔 材料制备方面的应用引起了研究者的关注. 本文将 重点介绍 $\mathrm{ScCO}_{2}$ 在分子篮、碳纳米管、金属/复合氧化 物纳米粒子、金属有机骨架材料(MOF)和聚合物等多 孔材料合成方面研究的最新进展.

\section{$1 \mathrm{ScCO}_{2}$ 在分子篮合成中的应用}

\section{$1.1 \mathrm{ScCO}_{2}$ 作为溶剂用于分子篮合成和改性}

在分子篮的改性中, 溶剂的选择至关重要, 这是 由于在溶剂携带反应物进人模板制备介孔材料时, 一般的有机溶剂经常会因为黏度太大而堵塞孔道, 使一些气态物质凝结在孔隙中, 进一步阻碍溶剂的 渗透; 而 $\mathrm{scCO}_{2}$ 具有气体的黏度以及高的质量传递速 率, 使得 $\mathrm{ScCO}_{2}$ 在分子篮改性中得到广泛应用. 
$\mathrm{Li}$ 等人 ${ }^{[2]}$ 以 $\mathrm{scCO}_{2}$ 作为载体/溶剂, 四氢呋喃作为 共溶剂, 制备了Al-MCM-41. 与传统的浸渍法和直 接水热合成法相比, 所制得的 Al-MCM-41具有更高 的水热稳定性和热稳定性. 对不同方法制得的 Al-MCM-41在 $373.15 \mathrm{~K}$ 下回流处理 $16 \mathrm{~h}$, 比较材料处 理前后的Brunauer-Emmett-Teller (BET)比表面积、孔 径、孔体积和 X射线衍射 (XRD) 峰的强度, 来衡量其 水热稳定性. 结果显示, 在 $\mathrm{scCO}_{2}$ 辅助下制得的 Al-MCM-41具有最好的水热稳定性, 直接水热合成 法制得的样品次之, 浸渍法制得的样品水热稳定性 最差. 比较不同方法制得的样品在高温㷽烧前后各 项指标的变化, 发现在 $\mathrm{scCO}_{2}$ 辅助下制得的 Al-MCM-41具有最好的热稳定性, 浸渍法制得的样 品次之, 直接水热合成法制得的样品热稳定性最差. 此外, 他们还发现, $\mathrm{scCO}_{2}$ 条件下铝原子能够较好地 扩散到孔壁的表面, 在不破坏介孔结构的情况下, 形 成硅铝酸盐层, 这对该材料应用到催化领域中具有 积极意义.

Sun等人 ${ }^{[3]}$ 在 $\mathrm{scCO}_{2}$ 条件下使用表面凝胶-溶胶修 饰 SBA-15, 在其表面嫁接单层和双层 $\mathrm{TiO}_{2}$, 形成 $\mathrm{TiO}_{2}$ 的金属氧化物薄膜. 覆盖上的 $\mathrm{TiO}_{2}$ 主要以锐钛 矿的形式存在, 氮气吸附实验表明, 随着覆盖次数的 增加, 比表面积和孔尺寸减小, 最终可得厚度为 $5 \AA$ 的 $\mathrm{TiO}_{2}$ 薄膜. Feng等人 ${ }^{[4]}$ 在 $\mathrm{scCO}_{2}$-乙醇溶液中将 $\mathrm{Ti}$ 掺 杂人 SBA-15制得了 Ti-SBA-15. $\mathrm{scCO}_{2}$ 优异的溶解性 能可以在不堵塞SBA-15分子篎的孔道情况下, 使金 属前驱体转移至孔道中生成纳米粒子.

Wang 等人 ${ }^{[5]}$ 使用含仲丁醇铝的 $\mathrm{scCO}_{2}$ 对合成的 MCM-48分子篮进行后处理, 并对样品进行水热稳定 性的考察, 发现超临界条件下处理过的MCM-48水热 稳定性高于未经处理的样品. 水热稳定性的提高主 要是由于前驱体 $\mathrm{Al}$ 和 $\mathrm{scCO}_{2}$ 两者的协同作用, 并且 $\mathrm{Al}$ 在后处理中具有重要作用, 因为仅用 $\mathrm{scCO}_{2}$ 处理或者 使用 $\mathrm{Zr}$ 和 $\mathrm{Ti}$ 代替 $\mathrm{Al}$ 作前驱体制得的 MCM-48的水热 稳定性没有明显的提高. 他们认为 $\mathrm{scCO}_{2}$ 的高渗透能 力, 使得 $\mathrm{Al}$ 原子进入 $\mathrm{MCM}-48$, 并形成比 $\mathrm{Si}-\mathrm{O}-\mathrm{Si}$ 键 更稳定的 $\mathrm{Al}-\mathrm{O}-\mathrm{Si}$ 键, 从而提高了 $\mathrm{MCM}-48$ 的水热稳 定性.

\section{$1.2 \mathrm{scCO}_{2}$ 用作清除剂}

合成分子篮时, $\mathrm{ScCO}_{2}$ 可以用来脱除作为模板剂 的表面活性剂. 清除模板剂的传统方法主要是煅烧,
煅烧可能改变或者破坏分子篮原有的孔型和孔径, 而使用超临界流体清除模板剂则能避免对分子篮结 构的影响.

Huang等人 ${ }^{[6]}$ 发现使用掺人甲醇的 $\mathrm{scCO}_{2}$ 对新合 成的多种介孔硅材料进行处理, 在 $1 \mathrm{~h}$ 内能够移除最 高达 $96 \%$ 的胺型表面活性剂, 而使用液态乙醇只能 萃取 $78 \%$ 的表面活性剂. 通过XRD和BET分析发现, 相比液态乙醇萃取方法或煅烧方法处理的分子篎, 使用超临界萃取方法处理的分子篮, 其结构保持较 好, 并且具有更高的水热稳定性和热稳定性.

使用超临界萃取的方法去除模板剂, 不仅能够 较好保持分子篮的结构, 而且具有清除率高的优点. Huang等人 ${ }^{[7]}$ 采用超临界流体萃取的方法处理合成后 的SBA-1, SBA-3, MCM-41和MCM-48, 有机模板剂 去除率超过 $80 \%$. Chatterjee等人 ${ }^{[8]}$ 采用掺人甲醇的 $\mathrm{scCO}_{2}$ 在压力 $35 \mathrm{MPa}$, 温度 333,353 和 $393 \mathrm{~K}$ 时进行萃 取, 结果显示, 当 $\mathrm{Si} / \mathrm{Ti}$ 为 200 (摩尔比), 温度为 $353 \mathrm{~K}$ 时, 萃取率达到最高为 $97.62 \%$.

研究发现, 使用 $\mathrm{scCO}_{2}$ 萃取去除表面活性剂的过 程具有可逆性 ${ }^{[9]}$. 研究者使用掺人甲醇或水的 $\mathrm{scCO}_{2}$ 作为清除剂, 研究了分子篮孔径大小对超临界流体 萃取后的模板剂率的影响. 结果表明, 孔径较大的介 孔材料(MCM-48)的模板剂被脱除后, 复原率可高达 $80 \%$; 而孔径较小的微孔材料(MFI和Beta分子篮)的 模板剂在脱除后, 复原率很低. 进一步研究发现, 萃 取的压力、温度和萃取剂的流速, 对表面活性剂的复 原有着重要影响.

\section{$1.3 \mathrm{ScCO}_{2}$ 辅助高聚物为模板制备分子篎膜}

高聚物几乎不溶于 $\mathrm{scCO}_{2}$, 而许多小分子物质与 $\mathrm{scCO}_{2}$ 相容性好, 因此, 高聚物可以作为模板剂, 同 时, 制备成分子篎膜后, 能在不破坏模板的情况下解 吸 $\mathrm{CO}_{2}$. $\mathrm{Pai}$ 等人 ${ }^{[10]}$ 以 2 种高分子材料 (共聚物 PEOb-PPO-b-PEO和PE-b-PEO)作为模板, 将两类模板先 在333.15 K退火, 然后在333.15 K和12.3 MPa下, 通 人含硅酸四乙酯的 $\mathrm{scCO}_{2}$ 溶液中保持 $2 \mathrm{~h}$. 最后, 在大 气环境下 $673.15 \mathrm{~K}$ 煅烧 $6 \mathrm{~h}$ 以除去模板, 可得到中孔 的分子篮薄膜.

除了以上几种用途, 朱国巍 ${ }^{[11]}$ 研究了 $\mathrm{scCO}_{2}$ 对制 备微孔TS-1分子篮的影响. 结果发现, $\mathrm{scCO}_{2}$ 压力为 $15 \mathrm{MPa}$ 下制备的 TS-1 晶化 $24 \mathrm{~h}$ 就能达到传统方法晶 化72 $\mathrm{h}$ 的结晶度. 推测这是因为碳酸盐物种在 $\mathrm{scCO}_{2}$ 
中形成, 而 $\mathrm{scCO}_{2}$ 同时具有气液两相的优点, 有助于 MFI沸石结晶.

\section{$2 \mathrm{scCO}_{2}$ 在碳纳米管 $(\mathrm{CNTs})$ 合成中的应用}

\section{$2.1 \mathrm{scCO}_{2}$ 作为碳源合成CNTs}

目前广泛使用的合成CNTs的前驱体是石墨、甲 烷、乙炔、乙醇和乙烯等碳氢化合物. 相比于这些原 料, $\mathrm{CO}_{2}$ 具有价格低和来源广等优点, 因此受到研究 者的关注. $\mathrm{Lou}$ 等人 ${ }^{[12]}$ 以 $\mathrm{CO}_{2}$ 作为碳源, 金属锂作为 还原剂合成了CNTs. 他们将固态干冰和金属锂加人 耐高压的不锈钢釜中, 在密闭条件下, 迅速升温至 $823.15 \mathrm{~K}$, 在压力为 $70 \mathrm{MPa}$ 下恒温 $10 \mathrm{~h}$, 自然冷却至 室温后进行后处理, 即可得到CNTs, 产率可以达到 $80 \%$ 左右. 在反应过程中, $\mathrm{CO}_{2}$ 既是反应物, 又是反 应体系. 与 $\mathrm{CO}_{2}$ 气体比较, $\mathrm{scCO}_{2}$ 能够增强 $\mathrm{CO}_{2}$ 在金属 锂表面的吸附, 从而促进电子传递过程. $\mathrm{Lou}^{[13]}$ 提出 了“金属纳米液滴”模型, 用来解释 $\mathrm{ScCO}_{2}$ 条件下, 金 属锂作为还原剂的CNTs的生成机理.

Vohs等人 ${ }^{[14]}$ 首次使用 $\mathrm{CCl}_{4}$ 作为前驱体, 在 $\mathrm{ScCO}_{2}$ 体系下, 合成了多壁碳纳米管(MWCNTs). $\mathrm{scCO}_{2}$ 在 合成CNTs时主要有 3 个作用: 清洗反应器, 除去水和 氧气; 辅助前驱体 $\mathrm{CCl}_{4}$ 的分解; 传输中间产物至金属 表面, 便于CNTs的成核和生长.

\section{$2.2 \mathrm{scCO}_{2}$ 用于CNTs的改性}

$\mathrm{Ye}$ 等人 ${ }^{[15]}$ 首次证实在 $\mathrm{scCO}_{2}$ 条件下, 金属纳米 粒子能够对 CNTs 进行均匀修饰. Lin等人 ${ }^{[16]}$ 使用 $\mathrm{ScCO}_{2}$ 将铂和钉以及两者的双金属合金均匀地涂布在 碳纳米管上, 得到了具有一定形貌特征的金属-碳纳 米管复合材料.

Sun等人 ${ }^{[17]}$ 研究发现, 以金属硝酸盐作为前驱 体, 在加人共溶剂乙醇的 $\mathrm{scCO}_{2}$ 中, 可以制备外表面 覆盖有金属氧化物膜 $\left(\mathrm{Ce}_{2} \mathrm{O}_{3}, \mathrm{CeO}_{2}, \mathrm{Al}_{2} \mathrm{O}_{3}\right.$ 和/或 $\left.\mathrm{La}_{2} \mathrm{O}_{3}\right)$ 的CNTs. 调节前驱体和CNTs的初始质量比, 可以调 控金属氧化物涂层的厚度. Sun等人 ${ }^{[18]}$ 进一步研究了 金属氧化物在 CNTs表面沉积的机理, 认为乙醇起溶 解前驱体的作用, 而 $\mathrm{scCO}_{2}$ 则辅助协调流体的性质. 通过控制反应过程, 实现了 CNTs 孔道内或孔道外 $\mathrm{SnO}_{2}$ 的选择性沉积. 通过对比有无 $\mathrm{CO}_{2}$ 的情况下进行 的相同沉积过程, 证实了 $\mathrm{scCO}_{2}$ 是金属氧化物微粒均 匀分布的关键所在. 无 $\mathrm{CO}_{2}$ 存在时, 只有很少的 $\mathrm{SnO}_{2}$
沉积在 CNTs 上, 而大量的 $\mathrm{SnO}_{2}$ 则发生了严重的团聚; 而 $\mathrm{ScCO}_{2}$ 独特的低黏度、低密度和表面张力几乎为零 的性质, 促使了 $\mathrm{SnO}_{2}$ 在 CNTs 上的均匀分布. Lock等 人 $^{[19]}$ 报道了在超临界条件下CNTs表面上聚合物的涂 布和孔道内的聚合物填充. Park等人 ${ }^{[20]}$ 在 $\mathrm{scCO}_{2}$ 环境 下浸渍有机金属化合物到单层碳纳米管-聚合物复合 物膜上, 合成了分散良好的金属修饰的纳米管-聚合 物复合材料. 他们发现超临界流体浸渍的过程能够 显著提高复合材料的韧性.

\section{$2.3 \mathrm{scCO}_{2}$ 干燥}

Seleznev等人 ${ }^{[21]}$ 以丙酮作为共溶剂使用 $\mathrm{scCO}_{2}$ 干 燥合成了独立式的半导体纳米管, 解决了干燥过程 中毛细作用力造成管坍塌的问题. Liu等人 ${ }^{[22]}$ 研究了 以丙醇作为共溶剂的 $\mathrm{scCO}_{2}$ 干燥 $\mathrm{CNT}$ 发射器的过程. 传统的热处理方法不能有效地除掉去离子水清洗后 CNT发射器中存在的残余水分, 而使其表现出较差 的场发射性能; 采用含 3\% 9\%(体积比)丙醇的 $\mathrm{scCO}_{2}$ 干燥法, 可以有效地去除水分, 激活 CNT发射器. 进 一步研究发现, 共溶剂丙醇对干燥过程起着极其重 要的作用, 它能提高水分子在疏水性的 $\mathrm{scCO}_{2}$ 下的溶 解度.

\section{$2.4 \mathrm{ScCO}_{2}$ 清洗}

Wang等人 ${ }^{[23]}$ 使用 $\mathrm{scCO}_{2}$ 作为溶剂除去了高压一 氧化碳合成工艺制备的单壁碳纳米管(SWCNT)中的 金属杂质. 具体过程如下: 首先, 使用大量含有乙二 胺四乙酸的电解质预处理SWCNTs; 然后, 采用原位 鳌合/超临界二氧化碳萃取去除金属颗粒; 最终在不 明显破坏纳米管的情况下，铁杂质移除率超过 $98 \%$.

\section{$3 \mathrm{ScCO}_{2}$ 在金属/复合氧化物纳米粒子制备 中的应用}

纳米粒子是指颗粒尺寸处于 1 100 $\mathrm{nm}$ 的粒子. 目前，由于表面效应和小尺寸效应而具有特异性质 的纳米粒子, 在化工、材料、治金、电子、日用化妆 品和生物医药等领域得到了广泛开发和应用. 金属/ 复合氧化物纳米粒子, 具有独特的晶体结构及表面 特性, 其催化活性和选择性大大高于传统催化剂, 是 催化研究领域一直寻找的高效催化材料. 制备纳米 粒子的传统方法主要有喷雾干燥、冷冻真空干燥、超 细粉碎、研磨等, 但这些方法存在产品溶剂残留 
高、粒度分布宽、生产成本高、生产时间长等问题. 超 临界流体微粒化技术可以很好地解决这些问题, 且 产品易分离, 洁净环保. 目前, 超临界流体微粒化技 术已成为制备和控制纳米粒子的一种有效技术 ${ }^{[24]}$, 在金属/复合金属氧化物催化剂制备的工业应用方面 表现出巨大的潜力.

教传琦等人 ${ }^{[25]}$ 对超临界流体快速膨胀法 (RESS)、超临界水热合成法、超临界干燥法、S纳米 粒子微乳液法等技术制备金属基纳米微粒做了总结. Zhang等人 ${ }^{[26]}$ 利用超临界 $\mathrm{CO}_{2} / \mathrm{H}_{2} \mathrm{O}$ 界面制备出了中空 的 $\mathrm{SiO}_{2}$ 和含钛 $\mathrm{SiO}_{2}$. Reverchon等人 ${ }^{[27]}$ 采用超临界抗 溶剂法 $(\mathrm{SAS})$ 制备出了平均直径 $50 \mathrm{~nm}$ 的 $\mathrm{ZnO}$ 前驱体 粒子, 粒子中存在的内孔使得BET比表面积达到了 $175 \mathrm{~m}^{2} / \mathrm{g}$, 显著提高了煅烧后氧化锌的催化活性.

近年来, 本课题组采用SAS法制备了一系列具 有介孔结构的金属氧化物和复合金属氧化物纳米颗 粒. 张金彦 ${ }^{[28]}$ 采用SAS法制备了平均孔径为 $6 \mathrm{~nm}$ 的 $\mathrm{CeO}_{2}$ 和铈锆复合氧化物前驱体, 发现 SAS法制备的 纳米铈锆固溶体颗粒具有分散均匀、尺寸小、形状规 则、储氧量高、热稳定性好等优点. Jiang等人 ${ }^{[29]}$ 在前 期工作的基础上优化了制备工艺条件, 使得铈锆氧 化物的比表面积、孔体积和储氧量均得到大幅提高. 同时, 研究发现所制备的铈锆氧化物具有中空结构, 作者还探索研究了SAS法制备的中空铈锆氧化物固 溶体纳米颗粒形成过程的机理.

本课题组 ${ }^{[30 ~ 34]}$ 采用 $\mathrm{CO}_{2}$ 为抗溶剂, 成功制备出 了介孔孔径的 Ce-Mn中空复合氧化物纳米粒子, 探 讨了中空纳米结构的形成机理, 发现前驱体溶液浓 度高低可控制中空结构的形成; 且通过调节SAS操 作参数可实现粒子的形貌、粒径和比例可控合成; SAS法所制备的中空纳米材料因具有较高的比表面 积而具有较好的储放氧性能和低温氧化还原性能, 有望成为良好的低温选择性催化脱硝反应和 $\mathrm{CO}$ 氧化 催化剂. Kuang等人 ${ }^{[35]}$ 和Huang等人 ${ }^{[36]}$ 采用SAS法制 备了介孔孔径的三元 $\mathrm{Cu}-\mathrm{Ce}-\mathrm{Zr}$ 复合氧化物和 $\mathrm{CeO}_{2}-\mathrm{ZrO}_{2}-\mathrm{Al}_{2} \mathrm{O}_{3}$, 进一步提高了材料的氧化还原性 能. 研究结果表明, $\mathrm{Cu}$ 的加人使得复合氧化物的储氧 量由506 $\mu \mathrm{mol} \mathrm{g}{ }^{-1}$ 增加到了 $636.9 \mu \mathrm{mol} \mathrm{g}{ }^{-1}$, 同时催化 剂的还原峰温度由 $873.15 \mathrm{~K}$ 左右降低到 393.15 513.15 K; 而 $\mathrm{Al}$ 的加人不仅提高了氧化物的 储放氧性能, 且提高了其抗烧结性能.

\section{$4 \mathrm{ScCO}_{2}$ 在MOF材料合成中的应用}

$\mathrm{MOF}$ 是近年来受到广泛关注的多孔材料, 在分 离、催化、气体存储等方面均得到广泛研究. 然而, 实 验测得的 MOF材料表面积小于基于 $\mathrm{X}$ 射线单晶解析 结构模拟得到的值, 并且不同研究者所报道的数据 也有差别 ${ }^{[37]}$. 这一现象表明, 传统的溶剂脱除过程 会造成孔道坞塌和溶剂滞留. 尤其是材料内部的介 孔极易受到材料不完全活化的影响, 在传统真空条 件下加热脱除溶剂处理后, 孔坍塌严重甚至介孔完 全丧失, 并且利用溶剂置换方法也并不能达到预期 效果.

针对上述问题, Hupp课题组 ${ }^{[38]}$ 报道了一种利用 $\mathrm{ScCO}_{2}$ 对 $\mathrm{MOF}$ 材料制备过程中残留溶剂进行脱除的 方法. MOF材料经过前期溶剂置换后, 在 $\mathrm{scCO}_{2}$ 条件 下处理 $30 \mathrm{~min}$, 然后慢慢排放 $\mathrm{CO}_{2}$. 结果表明 $\mathrm{scCO}_{2}$ 处理法不仅可以有效的减少材料孔道坞塌和孔道堵 塞, 使比表面积增加, 同时也可以防止材料颗粒聚 集. 另外, Hupp课题组 ${ }^{[39]}$ 还对MOF材料制备过程中 5 种残留溶剂的脱除方法进行了系统的比较: 真空热 处理法、溶剂置换法、 $\mathrm{ScCO}_{2}$ 处理法、冷冻干燥法和 化学处理法. 虽然这5种方法都能有效地提高MOF材 料的性能, 但 $\mathrm{ScCO}_{2}$ 活化法制备的 $\mathrm{MOF}$ 材料具有超强 表面性质, 且其无毒、造价低、原料易得等特性也使 得这种方法有很高的应用价值.

$\mathrm{CaO}$ 课题组 ${ }^{[40]}$ 用微波辅助溶剂热合成法与 $\mathrm{scCO}_{2}$ 活化法相结合制备了 $\mathrm{Cu}-\mathrm{BTC}$, 得到的材料有良好的 氮气吸附性能. Cu-BTC氢气吸附性能实验发现在温 度 $77 \mathrm{~K}$ 、压力 $18 \mathrm{bar}\left(1 \mathrm{bar}=10^{5} \mathrm{~Pa}\right)$ 时, 氢气吸附量达 到 $4.49 \%$ (质量百分比), 是当下报道中达到这一条件 下的最佳吸附量. 微波热溶剂法能够缩短结晶时间 而 $\mathrm{scCO}_{2}$ 活化法则能够得到较好的表面性质, 2种方法 相结合提供了一种快速有效的高储氢MOF材料的制 备方法. Cao课题组 ${ }^{[41]}$ 在 $\mathrm{Cu}-\mathrm{BTC}$ 的系统研究基础上, 对ZIF-8 与 MIL-53进行了 $\mathrm{scCO}_{2}$ 活化方法的研究. 将 处理后的 3 种材料与利用低沸点溶剂置换法处理的材 料进行对比. 通过小角XRD衍射(PXRD)、热重(TG)、 FT-IR、 $\mathrm{N}_{2}$ 吸附等手段对样品进行系统表征, 结果证 明, $\mathrm{scCO}_{2}$ 方法不仅能够比较彻底的脱除残留溶剂, 更能保证晶体骨架的完整性. $\mathrm{scCO}_{2}$ 方法处理过的材 料比表面积与孔容都较传统方法制备的材料有很大 的提高. 同时他们还证明了MOF材料的稳定性与活 
化方法并不相关.

在常规超临界活化方法的基础上, Koh等人 ${ }^{[42]}$ 提 出了流动的 $\mathrm{ScCO}_{2}$ 活化法. 这一方法是将样品放置于 塔内, 使 $\mathrm{scCO}_{2}$ 流动通过样品. 其优势在于可直接用 $\mathrm{ScCO}_{2}$ 来置换材料中残留的溶剂而不需要乙醇等媒 介. Zhao等人 ${ }^{[43]}$ 报道了一种用离子液体/超临界二氧 化碳/表面活性剂乳化系统制备MOF纳米微球的方 法. 该方法有效的将表面活性剂与适合溶剂相结合 制备理想MOF材料, 把利于传质的介孔与利于催化、 分离的微孔结构在MOF材料中结合了起来. 通过对 材料表征结果的讨论, 提出其合成机理为表面活性 剂自组装形成圆柱形胶束, 二氧化碳形成胶束内核, 而离子液体与有机分子形成连续相并在胶束上形成 孔洞. 这种方法为制备高性能MOF材料提供了一种 新的选择.

最近, Lopez-Periago 等人 ${ }^{[44]}$ 在不利用任何添加剂 和共溶剂的情况下仅使用 $\mathrm{scCO}_{2}$ 为溶剂制备了一维含 铜MOF材料. 实验证明, 利用超临界法合成MOF材 料关键在于反应物是否溶于 $\mathrm{scCO}_{2}$ 中, 该研究的反应 物均溶于 $\mathrm{scCO}_{2}$, 使得 $\mathrm{MOF}$ 的产率接近 $100 \% . \mathrm{scCO}_{2}$ 中一维 MOF材料的成功制备为更为复杂的MOF材料 的合成提供了有力的依据.

近年来, MOF材料作为载体负载纳米金属粒子 用于催化反应也是研究热点之一. 传统的制备方法 是采用化学气相沉淀法和浸渍法将金属前驱体置人 $\mathrm{MOF}$ 的孔道内, 经还原得到金属负载的 MOF材料 (M@MOF) 催化剂. 然而受到成本以及产品质量等条 件的制约, 新型制备方法亟待研究. 前面已介绍了利 用超临界流体技术制备碳纳米管负载的纳米金属催 化材料的研究. 研究者也发现利用超临界二氧化碳甲醇溶剂法可成功制备钉负载的MOF材料 ${ }^{[45]}$. 这种 $\mathrm{Ru} @ \mathrm{MOF}$ 材料对环己烯与苯的加氢反应有较好的催 化性能. 该方法制备过程简单, 且产品易从 $\mathrm{scCO}_{2}$ 分 离, 为金属负载MOF材料的制备提供了一种新的方 法. Wu等人 ${ }^{[46]}$ 对由该方法制备的Ru@Zr-MOF作了更 系统的研究, 将 $\mathrm{Ru}$ 固定在含 $\mathrm{Zr}$ 的MOF材料上, 并对 催化剂进行了系统的评价表征. 与传统浸渍方法相 比, 利用 $\mathrm{scCO}_{2}$ 制备的催化剂表面金属颗粒尺寸小、 分布均匀, 且在苯及其同系物加氢反应中催化剂活 性更高. Belyaeva等人 ${ }^{[47]}$ 首次采用 $\mathrm{scCO}_{2}$ 法制备了 $\mathrm{Pd} @ \mathrm{MOF}-5$ 催化剂. 结果表明采用 $\mathrm{scCO}_{2}$ 法合成的催 化剂, 金属离子多固定在MOF材料的孔道中, 有效
避免副反应的发生，从而提高了催化剂的选择性. 综 上所述, 利用 $\mathrm{scCO}_{2}$ 的诸多特性合成MOF及 $\mathrm{M} @ \mathrm{MOF}$ 催化材料已被证明拥有诸多优点, 亟待广大研究者 进一步探索.

\section{$5 \mathrm{ScCO}_{2}$ 在聚合物合成中的应用}

$\mathrm{scCO}_{2}$ 作为一种绿色环保溶剂, 因其所具有的廉 价无毒、环境友好等特点, 成为含孔道聚合物材料制 备过程的理想物理发泡剂. 微孔泡沫材料是利用高 惰性气体为发泡剂, 获得泡孔尺寸在 $10 \mu \mathrm{m}$ 以下、泡 孔密度在 $10^{9}$ cells $/ \mathrm{cm}^{3}$ 以上、具有闭孔结构的聚合物 材料. 利用 $\mathrm{scCO}_{2}$ 制备微孔泡沫最早在 1981 年由 Martini ${ }^{[48]}$ 提出. Cooper等人 ${ }^{[49]}$ 实现了在 $\mathrm{scCO}_{2}$ 中合成 交联聚合物小球, 与其他传统方法相比, $\mathrm{ScCO}_{2}$ 法得 到了直径小于 $0.5 \mu \mathrm{m}$ 的颗粒且收率达到 $95 \%$ 以上. 然 而，这一方法制备微孔聚合物的过程中，卸载压力时 反应体系温度仍处于聚合物玻璃化温度以上, 产生 不利于材料形成的表面张力, 故具有很大的局限性, 如孔道结构为微米孔且分布不均匀等. 龚剑亮和李 䂞 ${ }^{[50]}$ 在此方法的基础上开发了制备聚合物纳米多孔 材料的超临界选择溶胀法, 其原理是将纳米闭孔结 构加人聚合物材料中, 利用超临界二氧化碳对含亲 $\mathrm{CO}_{2}$ 组分嵌段聚合物的选择溶胀性, 对原合成方法进 行优化. 另外, 该方法能够获得非平衡态下的多种聚 合物纳米结构, 拓展了超临界流体在材料制备中的 应用. 新型方法的不断涌现，证明了 $\mathrm{scCO}_{2}$ 制备方法 对于孔道聚合物而言是一种理想、可调节、且值得广 泛研究的绿色制备方法.

除了利用 $\mathrm{scCO}_{2}$ 作为发泡剂生产微孔材料外, 可 将 $\mathrm{scCO}_{2}$ 作为聚合物非溶剂, 通过诱导聚合物溶液发 生相分离而制膜. 这一方法在 2001 年由 Kho等人 ${ }^{[51]}$ 提出. 诱导相分离制膜法可以对形成多孔膜起到干 燥作用, 同时避免后期干燥过程产生的结构坄塌问 题. 另外, 通过改变 $\mathrm{CO}_{2}$ 的温度和压力可实现对制膜 过程的可控调节.

另外，采用 $\mathrm{scCO}_{2}$ 可实现多孔材料表面积、孔隙 率、孔径的可控合成. Rouholamin等人 ${ }^{[52]}$ 系统研究了 聚乳酸微孔材料的合成条件对材料形貌特征的影响. 结果表明, 升高压力使得成核密度增加, 所得材料孔 尺寸小; 而延长 $\mathrm{CO}_{2}$ 卸压时间和饱和时间、升高温度 均会使孔尺寸变大孔壁变薄. 2015年, 牛首飞和杨文 玲 ${ }^{[53]}$ 对可降解聚乳酸多孔载体制备方法进行了研究, 
通过单因素实验以及正交实验得到聚乳酸多孔载体 最优SAS法生产工艺条件. 得到材料孔分布均匀, 具 有良好通透性的聚乳酸多孔载体.

另外, 基于二氧化碳在较温和条件下的相多变 性 ${ }^{[54]}$ (即临界条件较温和), 在合成聚合物多孔材料 时, $\mathrm{scCO}_{2}$ 可以同时充当分散相、转化媒介和聚合 点 ${ }^{[55]}$. 这对于减少工艺成本和环保等方面都有很强 的实际意义.

\section{6 结论与展望}

超临界 $\mathrm{CO}_{2}$ 稳定、无毒、环保, $\mathrm{CO}_{2}$ 来源广泛, 其 作为一种绿色溶剂已经在分子篮、碳纳米管、金属氧 化物和复合金属氧化物、MOF及聚合物等多孔材料 的合成方面展现出巨大的优势. 然而, 当前超临界 $\mathrm{CO}_{2}$ 辅助合成多孔材料的技术多处于实验室研究阶 段, 理论研究较少, 高通量合成多孔材料的方法有待 于进一步开发. 以超临界 $\mathrm{CO}_{2}$ 为介质的多孔材料合成
技术涉及到高压技术，需要较大的固定投资，同时， 合成过程中压力转换时需要较大的能量消耗, 因而 其操作费用较高, 这些因素限制了其在多孔材料合 成领域中更广泛的应用. 目前如何将其工业化, 还需 要进一步的探索和研究.

超临界流体由于其特殊的理化性质、稳定、无污 染等特点, 极大地迎合了现代化工工艺所追求的安 全、环保等理念, 在现代化学化工以及相关领域中表 现出了极好的应用前景. 尤其针对超临界流体技术 应用于材料制备过程, 其良好的性能已受到科学家 的广泛关注. 基于超临界流体技术环保、洁净易脱除 的特点, 其在食品、医药等方面有极好的应用潜质. 另外, 超临界 $\mathrm{CO}_{2}$ 临界条件温和, 能够很好地满足对 于热敏材料以及稳定性较差的材料的制备要求. 随 着对于超临界流体技术理论研究的进一步深人, 科 学界对于其在材料制备中的应用将有更深刻的理解, 这一技术将会在未来的生产中有更为广泛的应用.

\section{参考文献}

1 Han B X. Supercritical Fluid Science and Technology (in Chinese). Beijing: China Petrochemical Press Co. Ltd., 2005 [韩布兴. 超临界 流体科学与技术. 北京: 中国石化出版社, 2005]

2 Li S M, Xu Q, Chen J F, et al. Study and characterization of Al-MCM-41 prepared with the assistance of supercritical $\mathrm{CO}_{2}$. Ind Eng Chem Res, 2008, 47: 8211-8217

3 Sun D, Liu Z, He J, et al. Surface sol-gel modification of mesoporous silica molecular sieve SBA-15 with $\mathrm{TiO}_{2}$ in supercritical $\mathrm{CO}_{2}$. Micropor Mesopor Mater, 2005, 80: 165-171

4 Feng J, An G M, Chen B H, et al. Post-synthesis of Ti-SBA-15 in supercritical $\mathrm{CO}_{2}$-ethanol solution. Clean-Soil Air Water, 2009, 37: $527-533$

5 Wang K, Lin Y, Morris M, et al. Preparation of MCM-48 materials with enhanced hydrothermal stability. J Mater Chem, 2006, 16: 4051-4057

6 Huang Z, Xu L, Li J H, et al. Organic template removal from hexagonal mesoporous silica by means of methanol-enhanced $\mathrm{CO}_{2}$ extraction: Effect of temperature, pressure and flow rate. Sep Purif Technol, 2011, 77: 112-119

7 Huang Z, Huang L, Shen S, et al. High quality mesoporous materials prepared by supercritical fluid extraction: Effect of curing treatment on their structural stability. Microporous Mesoporous Mater, 2005, 80: 157-163

8 Chatterjee M, Hayashi H, Saito N. Role and effect of supercritical fluid extraction of template on the Ti(IV) active sites of Ti-MCM-41. Microporous Mesoporous Mater, 2003, 57: 143-155

9 Huang Z, Luan D, Shen S, et al. Supercritical fluid extraction of the organic template from synthesized porous materials: Effect of pore size. J Supercrit Fluid, 2005, 35: 40-48

10 Pai R A, Humayun R, Schulberg M T, et al. Mesoporous silicates prepared using preorganized templates in supercritical fluids. Science, 2004, 303: 507-510

11 Zhu G W. Synthesis and properties research of molecular sieves with aid of $\mathrm{CO}_{2}$ (in Chinese). Doctor Dissertation. Changchun: Jilin University, 2014 [朱国巍. $\mathrm{CO}_{2}$ 辅助合成分子篮及其性质研究. 博士学位论文. 长春: 吉林大学, 2014]

12 Lou Z S, Chen Q W, Wang W, et al. Synthesis of carbon nanotubes by reduction of carbon dioxide with metallic lithium. Carbon, 2003, 41: 3063-3067 
13 Lou Z S. Synthesis of carbon nanomaterials by reduction of carbon dioxide (in Chinese). Doctor Dissertation. Hefei: University of Science and Technology of China, 2005 [娄正松. 化学还原二氧化碳合成碳基纳米材料. 博士学位论文. 合肥: 中国科学技术大学, 2005]

14 Vohs J K, Brege J J, Raymond J E, et al. Low-temperature growth of carbon nanotubes from the catalytic decomposition of carbon tetrachloride. J Am Chem Soc, 2004, 126: 9936-9937

15 Ye X, Lin Y, Wang C, et al. Supercritical fluid fabrication of metal nanowires and nanorods templated by multiwalled carbon nanotubes. Adv Mater, 2003, 15: 316-319

16 Lin Y, Cui X, Yen C, et al. PtRu/carbon nanotube nanocomposite synthesized in supercritical fluid: A novel electrocatalyst for direct methanol fuel cells. Langmuir, 2005, 21: 11474-11479

17 Sun Z Y, Zhang X R, Han B X, et al. Coating carbon nanotubes with metal oxides in a supercritical carbon dioxide-ethanol solution. Carbon, 2007, 45: 2589-2596

18 Sun Z Y, Liu Z, Han B X, et al. Supercritical carbon dioxide-assisted deposition of tin oxide on carbon nanotubes. Mater Lett, 2007, 61: $4565-4568$

19 Lock E H, Merchan-Merchan W, D'Arcy J, et al. Coating of inner and outer carbon nanotube surfaces with polymers in supercritical $\mathrm{CO}_{2}$. J Phys Chem C, 2007, 111: 13655-13658

20 Park C, Kim J W, Sauti G, et al. Metallized nanotube polymer composites via supercritical fluid impregnation. J Polym Sci Pol Phys, 2012, 50: 394-402

21 Seleznev V, Yamaguchi H, Hirayama Y, et al. Single-turn GaAs/InAs nanotubes fabricated using the supercritical $\mathrm{CO}_{2}$ drying technique. Jpn J Appl Phys, 2003, 42: L791-L794

22 Liu P T, Tsai C T, Chang T C, et al. Activation of carbon nanotube emitters by using supercritical carbon dioxide fluids with propyl alcohol. Electrochem Solid State Lett, 2006, 9: 124-126

23 Wang J S, Wai C M, Shimizu K, et al. Purification of single-walled carbon nanotubes using a supercritical fluid extraction method. J Phys Chem C, 2007, 111: 13007-13012

24 Jung J, Perrut M. Particle design using supercritical fluids: Literature and patent survey. J Supercrit Fluid, 2001, 20: 179-219

25 Jiao C Q, Li H X, Liu B B. Present and prospect of supercritical fluid for preparing metal matrix nanoparticles (in Chinese). Mater Rev, 2011, 13: 119-123 [教传琦, 李宏煦, 刘彬涁. 超临界流体制备金属基纳米微粒的现状与展望. 材料导报, 2011, 13: 119-123]

26 Zhang $\mathrm{C}$, Zhang J, Zhang X, et al. Preparation of silica and titanium-containing silica hollow spheres at supercritical $\mathrm{CO}_{2} / \mathrm{H}_{2} \mathrm{O}$ interface. J Supercrit Fluid, 2007, 42: 142-149

27 Reverchon E, Della Porta G, Sannino D, et al. Supercritical antisolvent precipitation of nanoparticles of a zinc oxide precursor. Powder Technol, 1999, 102: 127-134

28 Zhang J Y. SAS preparation and characterization of nano-crystalline Ce-Zr-O catalyst (in Chinese). Master Dissertation. Tianjin: Tianjin University, 2008 [张金彦. 纳米晶铈锆复合氧化物催化剂的SAS法制备与表征. 硕士学位论文. 天津：天津大学, 2008]

29 Jiang $\mathrm{H}$, Huang $\mathrm{P}$, Liu L, et al. Controllable synthesis of $\mathrm{Ce}_{1-x} \mathrm{Zr}_{x} \mathrm{O}_{2}$ hollow nanospheres via supercritical anti-solvent precipitation. Mater Charact, 2012, 63: 98-104

30 Jiang D, Zhang M, Jiang H. Preparation and formation mechanism of nano-sized $\mathrm{MnO}_{x}-\mathrm{CeO}_{2}$ hollow spheres via supercritical anti-solvent technique. Mater Lett, 2011, 65: 1222-1225

31 Jiang D Y, Zhang M H, Li G X, et al. Preparation and evaluation of $\mathrm{MnO}_{x}-\mathrm{CeO}_{2}$ nanospheres via a green rout. Catal Commun, 2012, 17: 59-63

32 Zhang M, Jiang D, Jiang $\mathrm{H}$. Enhanced oxygen storage capacity of $\mathrm{Ce}_{0.88} \mathrm{Mn}_{0.12} \mathrm{O}_{y}$ compared to $\mathrm{CeO}_{2}$ : An experimental and theoretical in vestigation. Mater Res Bull, 2012, 47: 4006-4012

33 Jiang $\mathrm{H} \mathrm{X}$, Zhao J, Jiang D Y, et al. Hollow $\mathrm{MnO}_{x}-\mathrm{CeO}_{2}$ nanospheres prepared by a green route: A novel low-temperature $\mathrm{NH}_{3}-\mathrm{SCR}$ catalyst. Catal Lett, 2014, 144: 325-332

34 Wang H Q, Jiang H X, Kuang L, et al. Synthesis of highly dispersed $\mathrm{MnO}_{x}-\mathrm{CeO}_{2}$ nanospheres by surfactant-assisted supercritical antisolvent (SAS) technique: The important role of the surfactant. J Supercrit Fluid, 2014, 92: 84-92

35 Kuang L, Huang P, Sun H, et al. Preparation and characteristics of nano-crystalline $\mathrm{Cu}-\mathrm{Ce}-\mathrm{Zr}-\mathrm{O}$ composite oxides via a green route: Supercritical anti-solvent process. J Rare Earth, 2013, 31: 137-144

36 Huang $\mathrm{P}$, Jiang $\mathrm{H} \mathrm{X}$, Zhang $\mathrm{M} \mathrm{H}$. Structures and oxygen storage capacities of $\mathrm{CeO}_{2}-\mathrm{ZrO}_{2}-\mathrm{Al}_{2} \mathrm{O}_{3}$ ternary oxides prepared by a green route: Supercritical anti-solvent precipitation. J Rare Earth, 2012, 30: 524-528

37 Duren T, Milliange F, Ferey G, et al. Calculating geometric surface areas as a characterization tool for metal-organic frameworks. Phys Chem, 2007, 111: 15350-15356 
38 Nelson A P, Farha O K, Mulfort K L, et al. Supercritical processing as a route to high internal surface areas and permanent microporosity in metal-organic framework materials. J Am Chem Soc, 2008, 131: 458-460

39 Mondloch J E, Karagiaridi O, Farha O K, et al. Activation of metal-organic framework materials. CrystEngComm, 2013, 15: 9258-9264

40 Xiang Z H, Cao D P, Shao X H, et al. Facile preparation of high-capacity hydrogen storage metal-organic frameworks: A combination of microwave-assisted solvothermal synthesis and supercritical activation. Chem Eng Sci, 2010, 65: 3140-3146

$41 \mathrm{Hu}$ Z. The synthesis and performance of metal-organic framesorks and CNT@MOF composite (in Chinese). Master Dissertation. Beijing: Beijing University of Chemical Technology, 2011 [胡赞. 金属-有机骨架化合物及其碳纳米管杂化材料的合成与性能研究. 硕士学位 论文. 北京: 北京化工大学, 2011]

42 Koh K, Van Oosterhout J D, Roy S, et al. Exceptional surface area from coordination copolymers derived from two linear linkers of differing lengths. Chem Sci, 2012, 3: 2429-2432

43 Zhao Y J, Zhang J L, Han B X, et al. Metal-organic framework nanospheres with well-ordered mesopores synthesized in an ionic liquid/ $\mathrm{CO}_{2}$ /surfactant system. Angew Chem Int Ed, 2011, 50: 636-639

44 Lopez-Periago A, Vallcorba O, Frontera C, et al. Exploring a novel preparation method of 1D metal organic frameworks based on supercritical $\mathrm{CO}_{2}$. Dalton Trans, 2015, 44: 7548-7553

45 Zhao Y J, Zhang J L, Song J L, et al. Ru nanoparticles immobilized on metal-organic framework nanorods by supercritical $\mathrm{CO}_{2}$-methanol solution: Highly efficient catalyst. Green Chem, 2011, 13: 2078-2082

46 Wu T, Zhang P, Jun M, et al. Catalytic activity of immobilized Ru nanoparticles in a porous metal-organic framework using supercritical fluid. Chin J Catal, 2013, 34: 167-175

47 Belyaeva E V, Isaeva V I, Said-Galiev E E, et al. New method for catalyst preparation based on metal-organic framework MOF-5 for the partial hydrogenation of phenylacetylene. Russ Chem Bull, 2014, 63: 396-403

48 Martini J E. The production and analysis of microcellular foam. Master Dissertation. Boston: Massachusetts Institute of Technology, 1981

49 Cooper A I, Hems W P, Holmes A B. Synthesis of cross-linked polymer microspheres in supercritical carbon dioxide. Macromol Rapid Commun, 1998, 19: 353-357

50 Gong J L, Li L. Nanoporous polymer synthesis using supercritical fluid selective swelling method (in Chinese). Polym Bull, 2011, 6: 9-14 [龚剑亮, 李磊. 超临界流体选择溶胀法制备聚合物纳米多孔材料. 高分子通报, 2011, 6: 9-14]

51 Kho Y W, Kalika D S, Knutson B L. Precipitation of nylon 6 membranes using compressed carbon dioxide. Polymer, 2001, 42: 6119-6127

52 Rouholamin D, Smith P J, Ghassemieh E. Control of morphological properties of porous biodegradable scaffolds processed by supercritical $\mathrm{CO}_{2}$ foaming. J Mater Sci, 2013, 48: 3254-3263

53 Niu S F, Yang W L. Preparation of porous poly(lactic acid) supports by supercritical anti-solvent technology (in Chinese). Petrochem Technol, 2015, (1): 116-120 [牛首飞, 杨文玲. 超临界抗溶剂法制备聚乳酸多孔载体. 石油化工, 2015, (1): 116-120]

54 Picchioni F. Supercritical carbon dioxide and polymers: An interplay of science and technology. Polym Int, 2014, 63: 1394-1399

55 Boyere C, Leonard A F, Grignard B, et al. Synthesis of microsphere-loaded porous polymers by combining emulsion and dispersion polymerisations in supercritical carbon dioxide. Chem Commun, 2012, 48: 8356-8358 


\title{
Advances of supercritical $\mathrm{CO}_{2}$ application in porous materials synthesis
}

\author{
ZHANG MinHua $^{1,2}$, DONG XiuQin ${ }^{1,2}$ JIANG HaoXi $^{1,2}$, LÜ HuiSheng ${ }^{1,2}$ \& CHEN YiFei ${ }^{1,2}$ \\ ${ }^{1}$ Key Laboratory of Green Chemical Technology of Ministry of Education, Tianjin University R\&D Center of Petrochemical Technology, Tianjin \\ University, Tianjin 300072, China; \\ ${ }^{2}$ Collaborative Innovation Center of Chemical Science and Engineering, Tianjin 300072, China
}

Porous materials have attracted more and more attentions for variety of applications, such as gas adsorption and separation, water purification, catalysis, pharmaceutical, solar cells, supercapacitor, and sensors. Current methods for porous materials synthesis mostly use excessive organic solvent which is environmental unfriendly and need further wash or purifications. In contrast to the traditional method, supercritical fluid technology especially supercritical $\mathrm{CO}_{2}$, as a green process, is an attractive method for the synthesis of porous materials. This is because supercritical $\mathrm{CO}_{2}$ as a green solvent has many favorable properties, such as low-cost, abundant, non-toxic, non-flammable and easy to get. Furthermore, the fabrication process using supercritical $\mathrm{CO}_{2}$ is much more convenient as the process pressure and temperature could be easily controlled. In addition, the produced materials have better performance than the ones synthesized by other methods. Therefore, the utilization of supercritical $\mathrm{CO}_{2}$ is a promising method for porous materials synthesis in the future. In this article, the advances in the synthesis of zeolite, carbon nanotube, metal/composite metal oxide, metal organic frameworks and polymers using supercritical $\mathrm{CO}_{2}$ method were reviewed. In the synthesis of zeolite, solvent selection is very important. Conventional organic solvents would block the pores because of high viscosity and prevent further diffusion. Supercritical $\mathrm{CO}_{2}$ has low viscosity and high mass transfer rate and this could facilitate its applications in zeolite synthesis and modification. At the same time, the mild critical conditions and high resolution of supercritical $\mathrm{CO}_{2}$ make it as a suitable medium as the template and cleaner for zeolite and carbon nanotube preparation. For carbon nanotube fabrication, supercritical $\mathrm{CO}_{2}$ could also act as carbon source for the reaction. The metal/composite metal oxide prepared by supercritical $\mathrm{CO}_{2}$ technology has better catalytic performance. In metal-organic frameworks (MOFs) synthesis, traditional vacuum process for desolvation will make the framework collapse. Supercritical $\mathrm{CO}_{2}$ could remove the solvent in the framework under mild conditions while maintain the structure. As a result, the surface area, gas adsorption capacity, and catalytic performance of MOFs could be increased. Lastly, supercritical $\mathrm{CO}_{2}$ as foaming agent for polymers synthesis was reviewed.

supercritical $\mathrm{CO}_{2}$, porous materials, zeolite, metal organic framework, metal/composite metal oxide

doi: 10.1360/N972015-00305 